\title{
Intrinsic elastic conductors with internal buckled electron pathway for flexible electromagnetic interference shielding and tumor ablation
}

\author{
Wenqian $\mathrm{He}^{1}$, Rui Zhang ${ }^{2}$, Yuanyuan Cheng ${ }^{3}$, Chao Zhang ${ }^{4}$, Xiang Zhou ${ }^{4}$ Zhuangjian Liu ${ }^{5}$, \\ Xiaoyu $\mathrm{Hu}^{1}$, Zhongsheng Liu ${ }^{1}$, Jinkun Sun ${ }^{1}$, Yinsong Wang ${ }^{3}$, Dong Qian ${ }^{2}$ and Zunfeng Liu ${ }^{1 *}$
}

\begin{abstract}
The elastic conductor is crucial in wearable electronics and soft robotics. The ideal intrinsic elastic bulk conductors show uniform three-dimensional conductive networks and stable resistance during large stretch. A challenge is that the variation of resistance is high under deformation due to disconnection of conductive pathway for bulk elastic conductors. Our strategy is to introduce buckled structure into the conductive network, by self-assembly of a carbon nanotube layer on the interconnecting micropore surface of a prestrained foam, followed by strain relaxation. Both unfolding of buckles and flattening of micropores contributed to the stability of the resistance under deformation $(2.0 \%$ resistance variation under $70 \%$ strain). Microstructural analysis and finite element analysis illustrated different patterns of two-dimensional buckling structures could be obtained due to the imperfections in the conductive layer. Applications as all-directional interconnects, stretchable electromagnetic interference shielding and electrothermal tumor ablation were demonstrated.
\end{abstract}

Keywords: intrinsic elastic conductor, buckled structure, threedimensional conductive network, electromagnetic interference shielding, electrothermal tumor ablation

\section{INTRODUCTION}

Stretchable electronic devices have been attractive for applications in soft robotics and wearable healthcare monitoring [1-3]. Elastic conductors play a key role in interconnecting these devices, or work as their electrodes, which can be divided into intrinsic and structural conductors. In general, conductive materials (such as metal nanowires and carbon-based nanomaterials) are rigid and non-stretchable, while soft elastomeric materials (such as polydimethylsiloxane (PDMS) and polyurethane (PU) sponge) are non-conductive. The fabrication of stretchable conductors has been realized successfully by combining conductive materials with soft substrates. However, such stretchable conductors did not have high stretchability, uniform bulk structure, and stable conductance. Therefore, intrinsic elastic conductors with high stretchability and small resistance change in low cost and easy processing are highly desirable in stretchable electronics, whereas they have not been developed yet.

The intrinsic conductors, such as solid metal/carbon filler elastomer composites [4,5], conductive copolymers or composites [6,7], and liquid metal (LM) composites $[8,9]$, show stretchability and conductivity. One efficient method is to disperse a conductive material into an elastic matrix to form a conductive network. For example, Baik's group [10] prepared a hybrid elastic film based on silver flakes and multi-walled carbon nanotubes (MWCNTs), and Takao's group [11] mixed silver flakes with fluorine rubber using fluorine surfactant to obtain good dispersion. Ko's group [12,13] demonstrated a highly stretchable and transparent conductor by embedding Ag nanowire percolation network on a PDMS film for elec-

\footnotetext{
${ }^{1}$ Key Laboratory of Functional Polymer Materials, College of Pharmacy, State Key Laboratory of Medicinal Chemical Biology, Nankai University, Tianjin 300071, China

${ }^{2}$ Department of Mechanical Engineering, University of Texas at Dallas, Richardson, TX 75080, USA

${ }^{3}$ Tianjin Key Laboratory on Technologies Enabling Development of Clinical Therapeutics and Diagnostics (Theranostics), School of Pharmacy, Tianjin Medical University, Tianjin 300070, China

${ }^{4}$ Jiangsu Key Laboratory of Bioactive Natural Product Research and State Key Laboratory of Natural Medicines, China Pharmaceutical University, Nanjing 210009, China

${ }^{5}$ Institute of High Performance Computing, A*STAR Research Entities, Singapore 138632, Singapore

* Corresponding author (email: liuzunfeng@nankai.edu.cn)
} 
tromagnetic interference (EMI) shielding and heating. Similarly, Bao's group $[14,15]$ prepared conductive polymer-based intrinsic conductors by mixing poly $(3,4-$ ethylenedioxythiophene) with elastomers or by copolymerization. These intrinsic elastic conductors show bulk structures and high conductivities, but the resistance change is high due to the decreased contact between the conductance pathway under stretching.

As an alternative, structural conductors show stable resistance change during stretch by use of buckled or serpentine structural design for rigid conductors [16-19]. Such structures are generally prepared by attaching the conductive thin layer on a pre-stretched elastomer substrate followed by stretch-release, or using digital mechanical cutter plotter. A wide range of materials have been used as the conductive layer, including metal nanowires [20,21], CNTs [22,23], and conductive polymers [24]. However, it is not feasible for the bi-layered structure to improve the conductivity by simply increasing the thickness of the conductive layer, because a thick, highmodulus top layer can hardly buckle on a low-modulus elastic substrate. When releasing the pre-strain, a soft elastic substrate would produce a compress strain on the top layer, whereas the compress strain is not enough to form the buckle in a thick, rigid conductive layer. Moreover, this bi-layered structure is difficult to use when an all-directional interconnect or a uniform bulk structure is required, such as in EMI shielding and radar wave absorption [25]. In our previous work [26], a layer- by-layer technique was used to prepare a multi-layer, buckled elastic conductor, which increased the conductivity while maintaining the resistance stability under deformation. However, this structure did not show conductivity in the thickness direction, and the preparation required complicate iterative coating-stretching processes. As such, it is still a challenge to obtain a bulk elastic conductor with constant resistance during deformation.

One possible solution is to introduce micron- or nanoscale buckled structures in the three-dimensional (3D) conductive network of the intrinsic conductors without using a substrate (Fig. 1a). A hot-pressing process can produce the buckled structure in the foam; when the conductive networks are partially broken during stretch, the resulting elastic conductor still shows a high resistance change ( $>20$ times resistance increase for $150 \%$ strain) [27]. To the best of our knowledge, this proposed configuration for obtaining stable resistance change has not yet been reported. In this paper, we successfully introduced the buckled structure into an intrinsic elastic conductor, using polypropylene (PP) foam as an elastic matrix and single-walled CNTs (SWCNTs) as the conductive phase. SWCNT formed a 3D network by selfassembly on the interconnecting pore surface of a prestretched PP foam; releasing the pre-stretch resulted in buckling of the SWCNT network. The resulting buckled SWCNT/PP foam showed $2.0 \%$ resistance change upon stretching for $70 \%$ strain, corresponding to a quality
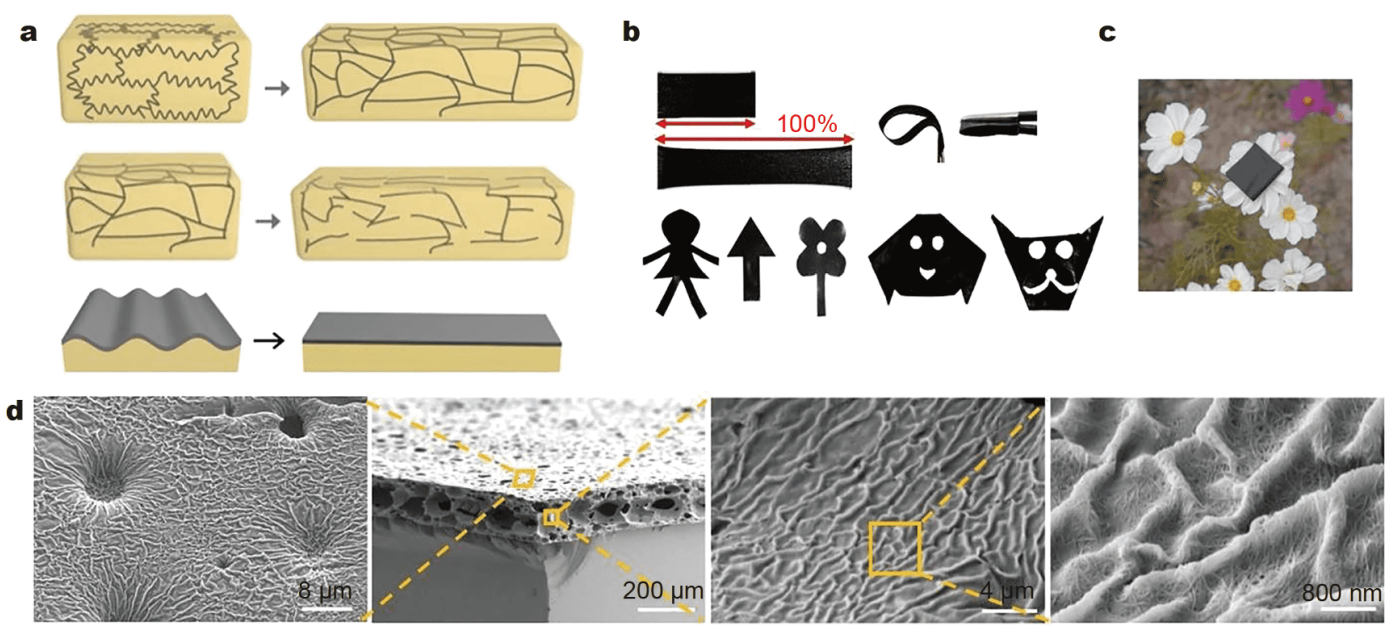

Figure 1 Structures of the intrinsic conductor with buckled 3D network. (a) Schematic demonstrations showing that (top) the buckled 3D conductive network in an intrinsic elastic conductor keeps intact during stretching, (middle) the 3D conductive network without buckled structure in an intrinsic elastic conductor disconnects during stretching, and (bottom) the elastic conductor with a buckled conductive surface layer keeps intact during stretching. (b) Optical photographs showing that the $\mathrm{BSPF}_{1}$ is stretched, bent, cut and folded into different shapes, indicating its excellent flexibility. (c) Optical photographs showing a piece of $\mathrm{BSPF}_{1}$ supported on the petal of a primula flower, indicating its light weight. (d) Low and high resolution SEM images for biaxial $\mathrm{BSPF}_{3}$ observed from the surface and the cross-section. The $\mathrm{BSPF}_{m}$ in (b) to (d) were prepared by using biaxial strain of $35 \%$. 
factor $\left(Q=\left(\Delta R / R_{0}\right) /\left(\Delta L / L_{0}\right) . \Delta R\right.$ and $R_{0}$ represent the resistance change and initial resistance of the sample; $\Delta L$ and $L_{0}$ represent the length change and initial length) of 0.028 , which is much lower than those of other state-ofthe-art intrinsic conductors. We also showed their potential applications in all-directional electrical interconnects, EMI shielding, and tumor ablation.

\section{EXPERIMENTAL SECTION}

\section{Fabrication of the SWCNT/PVP ink}

SWCNTs (average length of $\sim 5 \mu \mathrm{m}$ and diameter of $1.6 \mathrm{~nm}$, OCSiAl TUBALL ${ }^{\mathrm{TM}}$ Co. Ltd.) was mixed with sodium dodecyl sulfate (SDS, $0.1 \mathrm{wt} \%$, Sigma-Aldrich Co.) in deionized water and dispersed with high-shear mixing (Cat: X120), followed by sufficient sonification to ensure dispersion of SWCNTs in water. Polyvinylpyrrolidone (PVP, Lot no.P48497-500g, Beijing Innochem Science Technology Co. Ltd.) was then added, followed by $12 \mathrm{~h}$ sonification at $50^{\circ} \mathrm{C}$ [28]. The weight percents of the SWCNT and PVP are $0.2 \%$ and $0.4 \%$, respectively.

\section{Fabrication of $\mathbf{B S P F}_{m}$}

The PP foam with a thickness of $150 \mu \mathrm{m}$ purchased from Nitto Denko Co. Ltd. (Model 0924512) was pre-stretched uni-axially or bi-axially to different strains and fixed on a home-made frame with the use of parafilm. It was then immersed in the SWCNT/PVP ink for $10 \mathrm{~min}$, and then dried at $50^{\circ} \mathrm{C}$ in an oven for $5 \mathrm{~min}$ to evaporate water. The above processes were repeated for a certain number of cycles to increase the SWCNT loading, and then the composite foam was relaxed from the frame to form $\mathrm{BSPF}_{m}$ ( $m$ represents the number of cycles repeated). If not specified, the frame used for biaxial stretch had a dimension of $6 \mathrm{~cm} \times 6 \mathrm{~cm}$, and the frame used for uniaxial stretch had a dimension of $4 \mathrm{~cm} \times 9 \mathrm{~cm}$.

\section{Characterizations}

Raman spectra were conducted on a Labram spectrometer (SR-500I-A) with laser wavelength of $532 \mathrm{~nm}$. The scanning electron microscopy (SEM) images were taken on a field emission scanning electron microscope (MERLIN Compact). The transmission electron microscopy (TEM) images were obtained on a transmission electron microscope (model JEM-2800). The SEM images were obtained as follows. First, the $\mathrm{BSPF}_{1}$ was stretched to different strains $(0 \%, 30 \%$, and $50 \%)$, the both ends were fixed by using copper wires, and the SEM images were obtained by attaching the $\mathrm{BSPF}_{1}$ vertically on the silicon wafer to expose the cross-section of the foam to the electron beam. It should be emphasized that these SEM images at different strains originated from the same position. The electrical resistances of the samples were measured on a Keithley SourceMeter (2400) using a fourprobe configuration. The electrical resistance measurements were repeated for five times to obtain the average value. The mechanical properties were obtained on an Instron mechanical tester (model 3365), with a $5 \mathrm{~N}$ load cell and at a stretch rate of $60 \mathrm{~mm} \mathrm{~min}^{-1}$. The stress and strain were engineering values. The contents of SWCNT in $\mathrm{BSPF}_{m}$ were obtained by thermogravimetric analysis (TGA) on a thermogravimetric analyzer (model TGA/ DSC1), under air atmosphere at a heating rate of $10^{\circ} \mathrm{C} \mathrm{min}{ }^{-1}$ from room temperature to $600^{\circ} \mathrm{C}$. Thermal infrared (IR) images and temperature were obtained on an infrared thermometer (FLIR T440). The EMI shielding effectiveness (SE) was obtained on a vector network analyzer (model Agilent N5242A) in a frequency range of 8-12 GHz. The sample size was $22.5 \mathrm{~mm} \times 10.0 \mathrm{~mm} \times$ $2 \mathrm{~mm}$. Micro computed tomography was carried out on Cytovia (model HSI 3D Plus).

\section{RESULTS AND DISCUSSION}

\section{Fabrication and morphology of buckled SWCNT/PP foam}

The SWCNTs used in this work had an average diameter of $1.6 \mathrm{~nm}$ and a length of $5 \mu \mathrm{m}$ (aspect ratio $>3000$ ), so it showed a high flexibility. The Raman spectra of SWCNTs showed a high G-band peak at $1590 \mathrm{~cm}^{-1}$ and a small Dband peak at $1330 \mathrm{~cm}^{-1}$ (Fig. S1a), indicating a highly graphitic structure. The SWCNTs $(0.2 \mathrm{wt} \%)$ were dispersed in water by using SDS $(0.1 \mathrm{wt} \%)$ and PVP (0.4 wt\%) as dispersants [28], which formed a highly sticky ink and did not show sedimentation for over three months (Fig. S1b). The TEM images showed the coating of SWCNTs by PVP chains (Figs S3 and S4). The PP foam used in this paper contained $90 \%$ porosity with interconnecting pores with an average pore size of $24 \mu \mathrm{m}$ (Figs S5 and S6a). The large porosity and interconnecting pores enabled effective penetration of CNTs inside the foam. On the other hand, the pore size and distribution also affected the stretchability of the PP foam. Increasing pore size or distribution could share more applied strain under the foam, which is beneficial to stabilizing the deformation. The PP foam showed good hydrophilicity (Fig. S6b and Movie S1), enabling the wetting of SWCNT/PVP water dispersion and conductive network formation upon drying.

Dipping the PP foam directly in SWCNT dispersion for 
$10 \mathrm{~min}$, followed by drying in an oven at $50^{\circ} \mathrm{C}$ for $5 \mathrm{~min}$ to evaporate the water, made the CNTs form an interconnecting conductive network. Such a conductive network did not contain the internal buckled structure, so the PP foam was pre-stretched before being dipped into the SWCNT dispersion in the following experiments. This PP foam can be reversibly extended to as large as $35 \%$ strain during the biaxial stretch or can be reversibly extended by up to $80 \%$ for uniaxial stretch. Consistent with the above method, releasing a biaxial pre-strain after drying out enabled the formation of buckles in the SWCNT conductive network (Fig. S2). But it should be noted that the foam swelled by $3 \%$ lateral strain by being dipped into the SWCNT dispersion for $10 \mathrm{~min}$. The PP foam could recover the initial size when it was dried. SEM images showed the bi-directional buckled SWCNT layer on the pore surface inside the foam (Fig. 1d). The crosssectional images indicated that no delamination of SWCNT occurred from the pore surface (Fig. S7). Micro computed tomography showed a 3D-connected network of conductive layer with buckled structure (Movie S2). Optical pictures showed that the buckled SWCNT/PP foam was light-weight and could stand on the petal of a primula flower (Fig. 1c). It could tolerate various mechanical deformations such as stretching and bending, and could be easily cut and folded into different shapes (Fig. 1b). Similarly, the uniaxial buckled SWCNT conductive network was also prepared by using uniaxial prestretch of PP foam.

It should be mentioned that, for biaxial pre-stretch, the same pre-strain in both directions were used (called fabrication strain, $\left.\varepsilon_{\text {fab }}\right)$. For increasing the conductivity, the pre-stretched foam was dip-coated with SWCNT ink and dried for seven cycles before the release of pre-strain, and $\mathrm{BSPF}_{m}$ was used to represent the buckled SWCNT/PP foam with $m$ dip-coating cycles.

\section{Electrical and mechanical properties of BSPF $_{m}$}

In order to qualitatively test the all-directional bulk conductivity and stretchability of the $\mathrm{BSPF}_{m}$, we connected the light emitting diodes (LEDs) from the top surface to the bottom surface, or to the side surface, or to the freshly-cut surface of the $\mathrm{BSPF}_{1}(1.2-\mathrm{cm}$-thick) using copper wires, and the LEDs came to light (Fig. S8a-c). This indicates the bulk conductivity of $\mathrm{BSPF}_{m}$. In addition, a 4 -mm-thick $\mathrm{BSPF}_{3}$ was used as a stretchable interconnect for a clock, by connecting the copper wires to its different surfaces. The clock worked well when the $\mathrm{BSPF}_{3}$ was repeatedly stretched (Movie S3). We also connected six LEDs from the top and the bottom surfaces of four pieces of $\mathrm{BSPF}_{1}$, and attached them on the elbow of a person. The LEDs kept lighting up during the deformation of the elbow (Movie S4). Moreover, the brightness of the LEDs was negligibly changed by eye during various deformations such as twisting and coiling (Fig. S9).

In the following sections, we quantitatively investigated the electrical and mechanical properties of $\mathrm{BSPF}_{m}$, and their dependencies on the fabrication strain and number of dip-coating cycles for biaxial and uniaxial pre-stretches. For obtaining repeatable characterization results, the $\mathrm{BSPF}_{m}$ was pre-stretched for ten cycles to the maximum available strain before electrical and mechanical tests. All the tests were performed in uni-directional stretch.

Fig. 2a shows the percent resistance change $\left(\Delta R / R_{0}\right)$ as a function of strain for $\mathrm{BSPF}_{1}$ with different fabrication strains. The resistance increased under stretching, and returned to the initial value during stretch-release. With the increase in the biaxial fabrication strain from $0 \%$ to $35 \%$, the resistance change monotonically decreased from $15.1 \%$ to $3.8 \%$ for $70 \%$ uni-directional stretch. In more detail, a hysteresis in the reversible stretching test for a smaller biaxial fabrication strain was observed. This is because the buckle density increased with the increase in pre-strain (as shown from SEM images in Fig. S10), which prevents the conductive network from further stretching-based deformation and the increase in the resistance change for $\mathrm{BSPF}_{m}$. Hence, the hysteresis for larger fabrication strain decreased due to the structural integrity of the CNT network during deformation. Uniaxial mechanical tests showed that the $\mathrm{BSPF}_{1}$ can completely recover the initial mechanical properties five minutes after strain relaxation, indicating good mechanical reversibility (Fig. S11b).

The resistance per stretched length, $R_{\varepsilon} / L_{\varepsilon}$, also decreased with increasing fabrication strain (Fig. S11c), where $R_{\varepsilon}$ and $L_{\varepsilon}$ are the resistance and the length at strain $(\varepsilon)$, respectively. This should be ascribed to the increased SWCNT content in $\mathrm{BSPF}_{m}$ with higher fabrication strain (Fig. S11a), where an increased pore surface area could be coated with SWCNTs. This result indicates that higher fabrication strain helps obtain higher conductivity for $\mathrm{BSPF}_{m}$. Interestingly, the conductivity increased with increasing strain, corresponding to the decreased $R_{\varepsilon} / L_{\varepsilon}$ at a higher strain level (Fig. S11d).

We then investigated the dependencies of electrical and mechanical properties on the number of dip-coating cycles for preparing the $\mathrm{BSPF}_{m}$. With increasing $m$ from one to seven, SWCNT contents increased and correspondingly the conductivity at the relaxed state increased from 

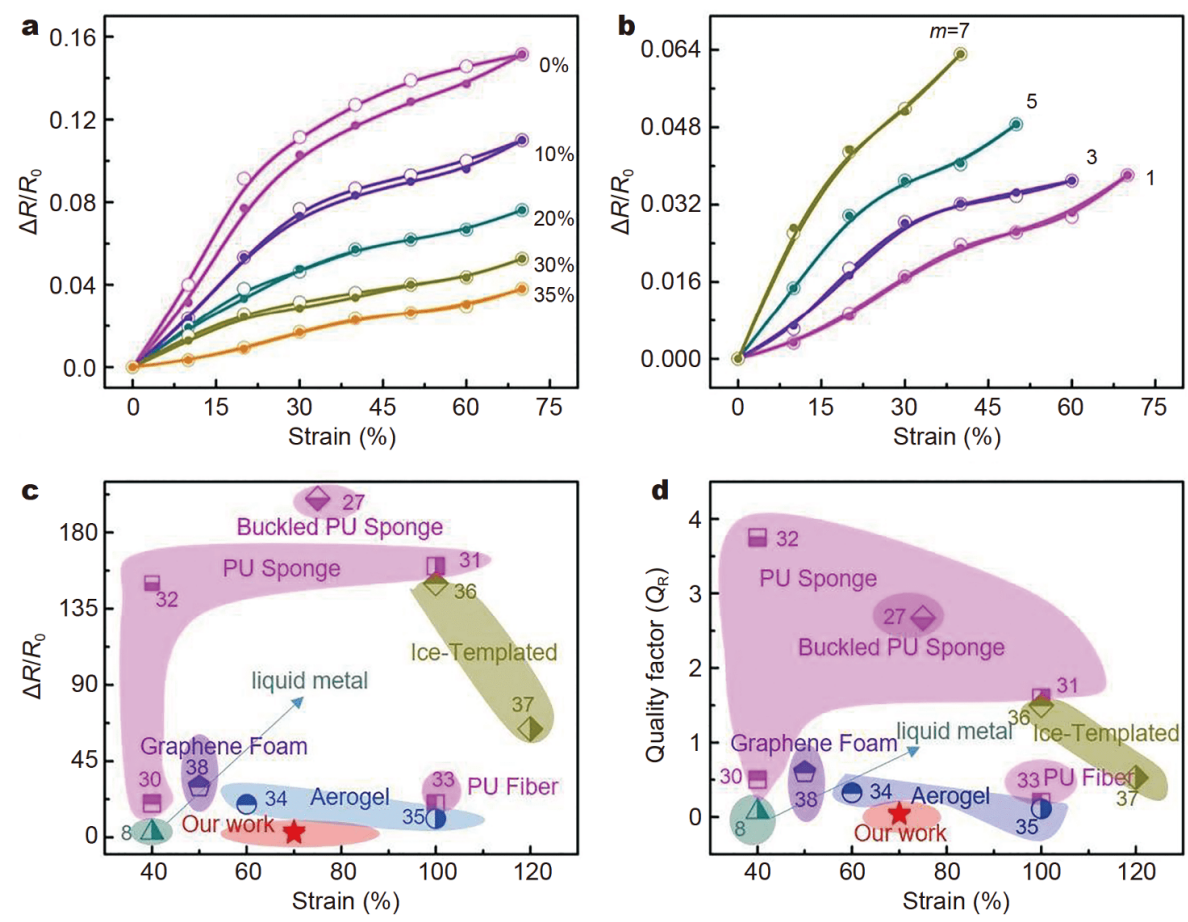

Figure 2 Stability of resistance for $\mathrm{BSPF}_{m}$ at different applied strains. (a) $\Delta R / R_{0}$ as a function of the applied strain for $\mathrm{BSPF}_{1} \mathrm{with}_{\text {different biaxial }}$ fabrication strains. (b) $\Delta R / R_{0}$ as a function of the applied strain for $\mathrm{BSPF}_{m}$ with $m$. (c, d) Comparison of (c) resistance change and (d) quality factor with maximum available strain for $\mathrm{BSPF}_{m}$ with previously reported typical 3D intrinsic stretchable bulk conductors. The filled and open symbols in (a) and (b) indicate loading and unloading processes, respectively.

5.2 to $60.6 \mathrm{~S} \mathrm{~m}^{-1}$ (Fig. S12a-c). The resistance change increased from $3.8 \%$ to $6.3 \%$ while the maximum available strain decreased from $70 \%$ to $40 \%$ (Fig. 2 b). This can be attributed to the increased rigidity of the conductive phase with increasing $m$ (Fig. S13a, b). Fig. S14 shows that the distance between the buckles of the SWCNT layer increased with increasing $m$. More importantly, $\mathrm{BSPF}_{m}$ showed good mechanical reversibility within the maximum available strain for different $m$ and multiple stretch-release cycles (Fig. S13c, d), which would facilitate their use as elastic interconnects.

In order to show the generality of this pre-stretch-based approach for buckle formation in intrinsic elastic conductors, we further investigated the $\mathrm{BSPF}_{m}$ with uniaxial fabrication strain. The uniaxial $\mathrm{BSPF}_{m}$ presented parallel buckled structures perpendicular to the pre-stretch direction, which showed increased a buckle density with increasing fabrication strain and decreased buckle period with increasing $m$ (Figs S15 and S16). Similar dependencies of electrical and mechanical properties on strain were obtained for uniaxial $\mathrm{BSPF}_{m}$ with different fabrication strains and different numbers of dip-coating cycles (Fig. 3a and Figs S17-S19). For example, the resistance change decreased from $15.1 \%$ to $2.0 \%$ for uni- axial $\mathrm{BSPF}_{1}$ as the fabrication strain increased from $0 \%$ to $80 \%$. It is interesting that even when stretched beyond the fabrication strain, the uniaxial $\mathrm{BSPF}_{m}$ still showed relatively stable resistance. For instance, stretching the uniaxial $\mathrm{BSPF}_{1}$ with a fabrication strain of $50 \%$ to an applied strain of $70 \%$ only showed resistance change of $5.6 \%$ (Fig. 3a).

It is important for an intrinsic bulk conductor to show stable resistance when stretched in different directions. We then investigated the resistance change for stretching a biaxial $\mathrm{BSPF}_{1}$ and a uniaxial $\mathrm{BSPF}_{1}$ in different directions. Fig. $3 \mathrm{c}$ shows that no significant resistance difference was observed by stretching a biaxial $\mathrm{BSPF}_{1}$ to $70 \%$ strain along each of the pre-strain axis $(3.8 \%)$ or at $45^{\circ}$ direction (3.4\%) with the pre-strain axis. This resistance homogeneity in different axes is likely due to the fact that the bi-directional buckles in the SWCNT conducting network could accommodate deformations in different directions [29]. Different from the biaxial case, stretching the uniaxial $\mathrm{BSPF}_{1}$ in the pre-strain direction showed smaller resistance change $(2.0 \%)$ than along the $45^{\circ}$ direction $(3.6 \%)$ and in the perpendicular direction (5.5\%) (Fig. 3b). Nevertheless, then uniaxial $\mathrm{BSPF}_{m}$ stretched in different directions was more stable than the SWCNT/PP 

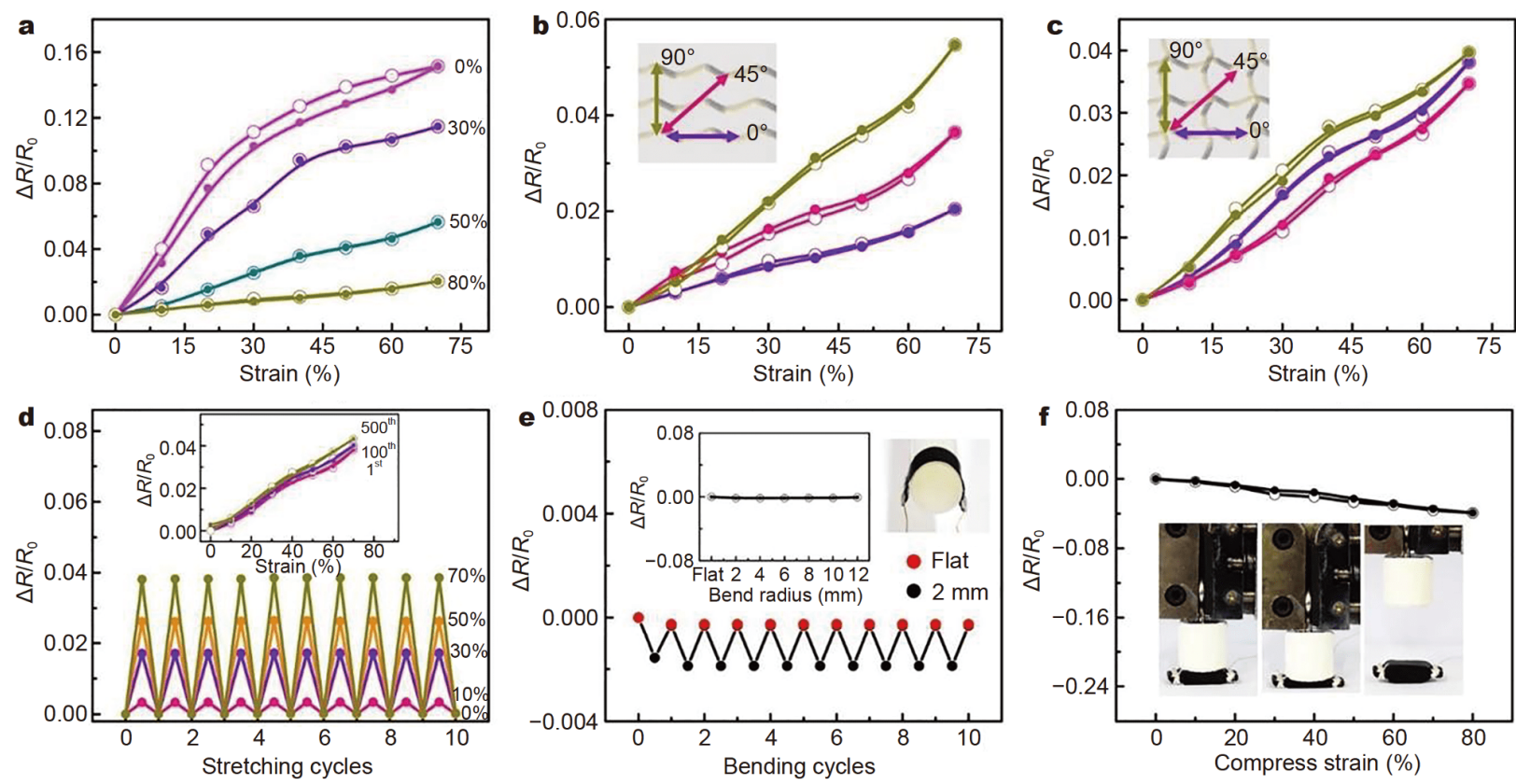

Figure 3 Electrical properties of $\mathrm{BSPF}_{m}$ for different types of deformations. (a) $\Delta R / R_{0}$ as a function of applied strain for BSPF ${ }_{1}$ with different uniaxial fabrication strains. (b, c) $\Delta R / R_{0}$ as a function of applied strain for (b) uniaxial BSPF ${ }_{1}$ and (c) biaxial BSPF $\mathrm{B}_{1}$ along different stretching directions. (d) $\Delta R /$ $R_{0}$ at different stretching/releasing cycles for biaxial BSPF${ }_{1}$. The inset shows the $\Delta R / R_{0}$ as a function of applied strain for the $1^{\text {st }}$, $100^{\text {th }}$, and $500^{\text {th }}$ cycles. (e) $\Delta R / R_{0}$ at different bending cycles for biaxial $\mathrm{BSPF}_{1}$ with a bending radius of $2 \mathrm{~mm}$. The left inset shows the $\Delta R / R_{0}$ as a function of bending radius, and the right inset shows an optical image of a BSPF ${ }_{1}$ bending around an 8-mm-diameter mandrel. (f) $\Delta R / R_{0}$ for biaxial BSPF as a function of applied compressive strain. The optical images from left to right in the inset show the $\mathrm{BSPF}_{1}$ under $30 \%$ and $70 \%$ compressions, and after relaxing compression, respectively. The filled and open symbols indicate loading and unloading processes, respectively. The uniaxial fabrication strain in (b) was $80 \%$, and the biaxial fabrication strain in $(\mathrm{c}-\mathrm{f})$ was $35 \%$.

foam without buckled structure (15.1\%). This indicates that $\mathrm{BSPF}_{m}$ is suitable for use as an intrinsic bulk conductor for different stretching directions.

To investigate the stability for long-term use, cycling tests were carried out on the biaxial $\mathrm{BSPF}_{m}$ for different types of deformations (Fig. 3d-f). The resistance negligibly changed by stretching the biaxial $\mathrm{BSPF}_{1}$ to different strains for ten stretch/release cycles, and slightly increased to $4.3 \%$ after 500 cycles, with the resistance at unloading slightly increased by $0.3 \%$. Bending the biaxial $\mathrm{BSPF}_{1}$ down to 2 -mm radius decreased the resistance by $0.16 \%$, which negligibly changed for ten cycles. Compressing the biaxial $\mathrm{BSPF}_{1}$ with $80 \%$ decrease in thickness resulted in a resistance change of $3.9 \%$, which negligibly changed for ten cycles (Fig. S20). Similar cycling results were obtained for uniaxial $\mathrm{BSPF}_{1}$ for stretching and bending deformations (Fig. S21).

Fig. $2 \mathrm{c}$ and $\mathrm{d}$ compare the percent resistance change and the quality factor $Q$ as a function of the maximum available strain for the $\mathrm{BSPF}_{m}$ with previous reported $3 \mathrm{D}$, bulk intrinsic elastic conductors [8,27,30-38], respectively. It can be seen that the uniaxial and biaxial $\mathrm{BSPF}_{m}$ showed the lowest percent resistance change and quality factor among these intrinsic conductors. Even if the maximum available strain $(70 \%)$ is not the highest number, it should meet the requirements for most cases of wearable electronic applications because this value is close to the maximum strain for person's movement (such as for elbow, 75\%) [39].

\section{Microstructural analysis during deformation}

In order to understand the mechanism of resistance stability during the deformation of the $\mathrm{BSPF}_{m}$, we here discussed the buckle formation in the interconnecting SWCNT network, and its microstructural evolution under deformation. The SWCNT/PVP film showed good conductivity, mechanical flexibility and toughness, which can be deformed into various shapes (Fig. 4a and c). The SWCNT/PVP film showed negligible resistance change upon stretching to $6 \%$. When coating on the pore surface of the PP foam, the SWCNT/PVP film formed an interconnecting conductive network inside the foam by selfassembly. An important point is that the SWCNT/PVP layer showed strong adhesion with the PP foam surface, 

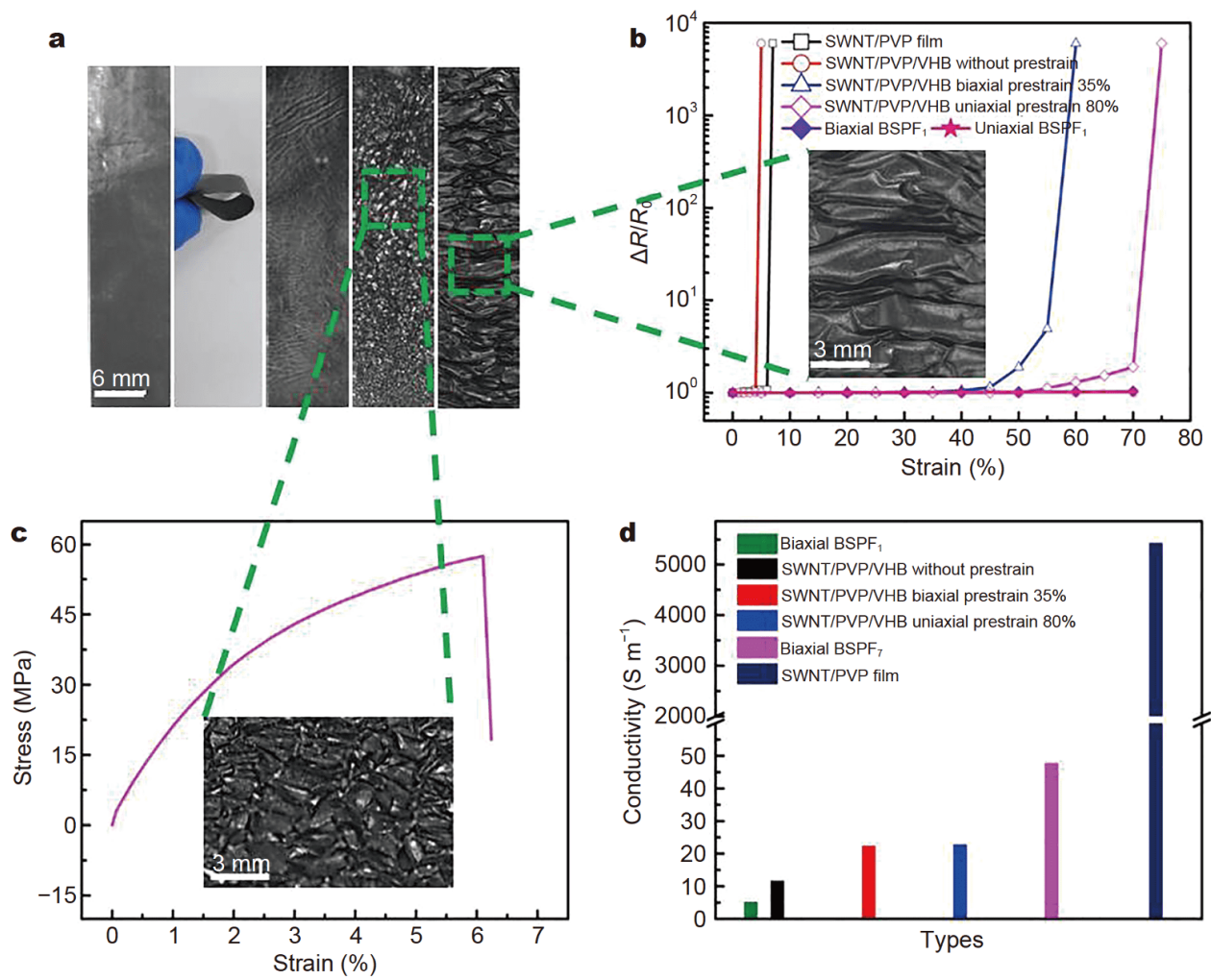

Figure 4 Optical images, electrical and mechanical properties of SWCNT/PVP film, SWCNT/PVP/VHB without pre-strain, SWCNT/PVP/VHB with uniaxial and biaxial fabrication strains. (a) Optical images from left to right were SWCNT/PVP film before and after bending, the SWCNT/PVP/VHB without pre-strain, the biaxial and uniaxial SWCNT/PVP/VHB. (b) $\Delta R / R_{0}$ as a function of applied strain for SWCNT/PVP film, SWCNT/PVP/VHB without pre-strain, SWCNT/PVP/VHB with biaxial fabrication strain of $35 \%$ and uniaxial fabrication strain of $80 \%$, and $\mathrm{BSPF}_{1}$ with biaxial fabrication strain of $35 \%$ and uniaxial fabrication strain of $80 \%$. (c) Stress-strain curve for SWCNT/PVP film. (d) Electrical conductivity as a function of applied strain for SWCNT/PVP film, SWCNT/PVP/VHB without pre-strain, SWCNT/PVP/VHB with biaxial fabrication strain of 35\%, SWCNT/PVP/VHB with uniaxial fabrication strain of $80 \%$, and $\mathrm{BSPF}_{1}$ and $\mathrm{BSPF}_{7}$ with biaxial fabrication strain of $35 \%$. For calculation of conductivity, the total thickness including VHB was considered.

so that no delamination occurred during the stretch-release-induced buckle formation (Fig. S7). In addition, the adhesion between two or multi- SWCNT/PVP layers is very strong due to the strong van der Waals forces between CNTs and PVP, as well as the entanglements between CNTs and PVP chains. Interestingly, the pore surface of PP foam also buckled along with the SWCNT/ PVP layer (Fig. S22). These properties endow the conductive network with good structural integrity during microstructural changes of the $\mathrm{BSPF}_{m}$.

Interestingly, we observed different morphologies for the biaxial buckled structures of the SWCNT conductive network (Fig. 5a-c). To understand the formation mechanism of these different types of morphologies, we performed finite element analysis (FEA) of the buckling of the SWCNT layer on the pores with bi-directional prestrain. A 3D representative volume element (RVE) model was established for a single pore that contained SWCNT/
PVP film on the PP substrate. Analysis showed that the buckle morphology of the film-on-pore substrate was highly sensitive to the imperfections of SWCNT layer during the release of the biaxial pre-strain. Similar structures were predicted as experimentally observed by choosing different imperfections in the conductive layer (Fig. 5d-f).

We next investigated the microstructural evolution of $\mathrm{BSPF}_{m}$ during deformation. A biaxial $\mathrm{BSPF}_{1}$ and a uniaxial $\mathrm{BSPF}_{1}$ were uniaxially stretched to different strains (30\% and $70 \%$ ), and the cross-sectional images along the stretching direction were obtained on SEM (Fig. $5 \mathrm{~g}-\mathrm{l}$ and Fig. S23). Under stretching, the parallel buckles in the uniaxial $\mathrm{BSPF}_{1}$ separated gradually, and interestingly the density of orthogonally interconnected buckles in both directions for the biaxial $\mathrm{BSPF}_{1}$ decreased. Wrinkles perpendicular to the stretching direction were observed for biaxial and uniaxial $\mathrm{BSPF}_{1}$, due to the Poisson's ratio 

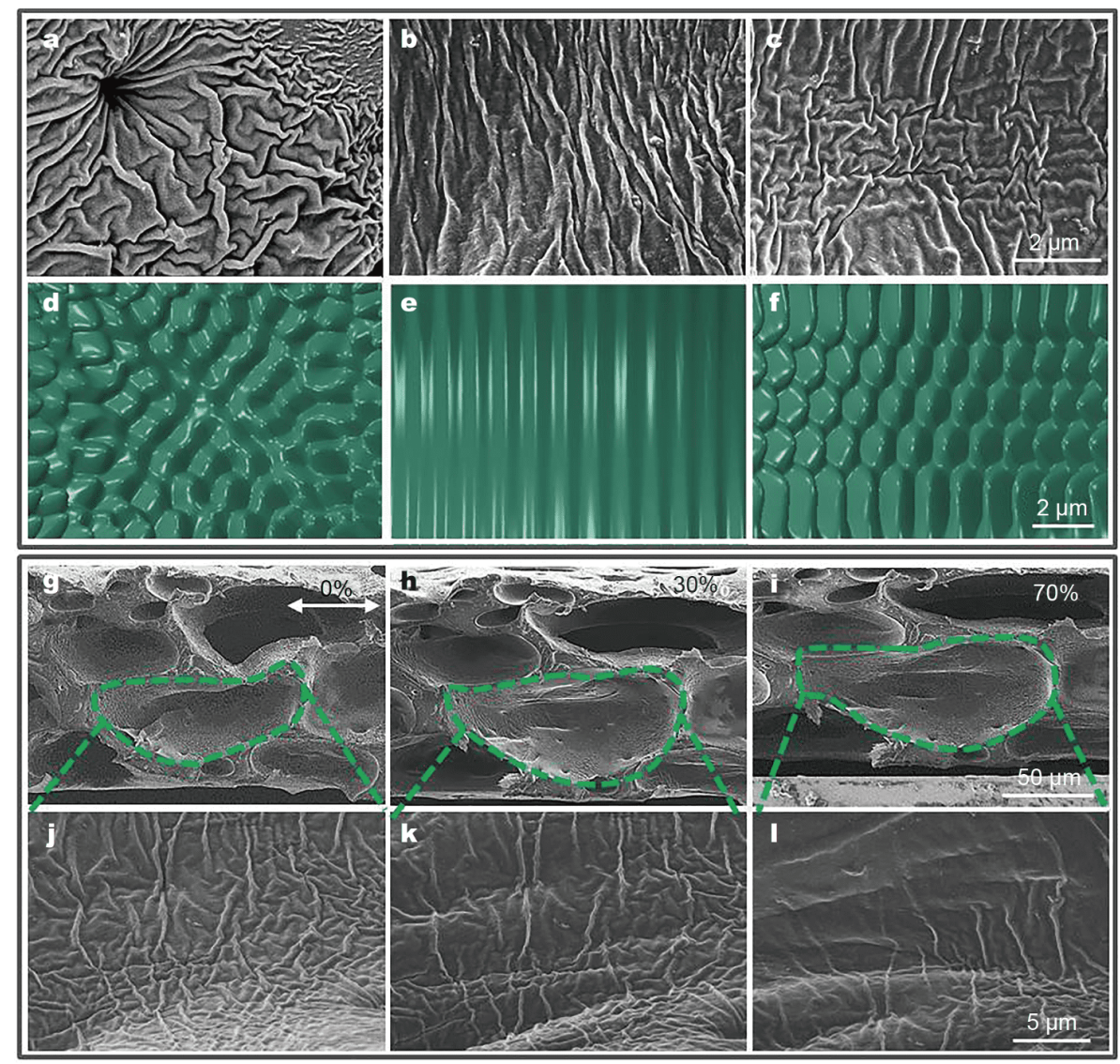

Figure 5 Microstructural analysis and FEA. (a-c) Different morphologies in SEM images for BSPF $_{1}$ with 35\% biaxial pre-strain. (d-f) Morphologies observed in FEA modeling similar to the experimental results in $(\mathrm{a}-\mathrm{c})$ for biaxial buckles by choosing different imperfections in the conductive layer. (g-l) SEM images for cross-sections of $35 \%$ biaxial pre-strain $\mathrm{BSPF}_{1}$ at applied strains of $0 \%, 30 \%$ and $70 \%$.

effect (size decrease in the direction perpendicular to the stretching direction) (Fig. S24).

The formation and unfolding of the buckles help maintain the SWCNT conductive network intact during stretch and keep resistance stable. As a comparison, we stretched a SWCNT/PP foam composite that was prepared without using pre-strain, and obvious cracks were observed by stretching to $70 \%$ strain, with SWCNTs being stretched out (Fig. S25). Note that the conductive layer was made by inter-connecting of 1D SWCNT bundles in the $2 \mathrm{D}$ surface. The re-organization of the SWCNT bundles that packed in the conductive layer might also partially accommodate the applied strain $[40,41]$. Furthermore, the micro pores were highly deformed and flattened under stretch, as shown in Fig. 5g-i and Fig. S25a-c, which can help to share the applied strain under the foam. This is another advantage of using interconnecting pores as a skeleton to construct the conductive network. For comparison, we prepared a
SWCNT/PVP film on VHB tape, a buckled SWCNT/PVP on 35\%-biaxially pre-strained $\mathrm{VHB}$, and a buckled SWCNT/PVP on $80 \%$-uniaxially pre-strained VHB, which showed dramatic increase $(>1000 \%)$ in resistance when stretched to $5 \%, 60 \%$, and $75 \%$ strains, respectively (Fig. 4b). Consequently, the flattening of pores, together with buckles unfolding, contributed importantly to the stable resistance of the interconnecting SWCNT network under large deformation.

\section{Applications of $\mathbf{B S P F}_{m}$}

\section{Stretchable EMI shielding}

With the development of wearable electronic devices, protection of human and devices that are sensitive to the electromagnetic pollution is highly desired. The $\mathrm{BSPF}_{m}$ showed highly stable resistance under large stretch (e.g., $2.0 \%$ and $3.8 \%$ of $\Delta R / R_{0}$ for uni- and biaxial $\mathrm{BSPF}_{1}$ at $70 \%$ of strain, respectively) and large content of micro pores 
( $90 \%$ porosity). This stretchable interconnecting conductive network would be a good candidate for EMI shielding under large deformation. Below we investigated the EMI SE at G-band $(8-12 \mathrm{GHz})$ for $\mathrm{BSPF}_{m}$ of $35 \%$ biaxial fabrication strain, as a function of SWCNT content and applied strain. If not specified, the $\mathrm{BSPF}_{m}$ composites had a thickness of $2 \mathrm{~mm}$. Fig. 6a shows that the average EMI SE increased from 11 to $45 \mathrm{~dB}$ with increasing the SWCNT contents from $3.8 \%(m=1)$ to $30.9 \%$ $(m=7)$.

We then investigated the EMI SE of the $\mathrm{BSPF}_{m}$ at different applied strains. Fig. $6 \mathrm{~b}$ shows that the average EMI $\mathrm{SE}$ only slightly decreased from 45 to $39 \mathrm{~dB}$ as the $\mathrm{BSPF}_{7}$ (with SWCNT content of $30.9 \mathrm{wt} \%$ ) was stretched from $0 \%$ to $30 \%$ strain. Detailed study about the reflection $\left(\mathrm{SE}_{\mathrm{R}}\right)$ and absorption $\left(\mathrm{SE}_{\mathrm{A}}\right)$ indicates that the electro- magnetic wave was largely absorbed rather than reflected by the $\mathrm{BSPF}_{m}$ (Fig. S26a, b). This is possibly because stretching caused flattening of micro pores, and then decreased the multi-reflection inside the foam $[42,43]$. Repeatedly stretching and releasing $\mathrm{BSPF}_{7}$ for 500 cycles up to $30 \%$ strain showed negligible decrease in EMI SE (Fig. 6c).

\section{Tumor ablation}

Thermal therapy of tumor is an important way for cancer treatment, in which the tumor tissues are subjected to a temperature higher than $45^{\circ} \mathrm{C}$ [44]. Photo-, magneto-, and electro-thermal tumor removal have been proved to be effective as an alternative to the direct tumor removal by surgery [45-47]. By considering that the $\mathrm{BSPF}_{m}$ showed a bulk conductivity and stable resistance during
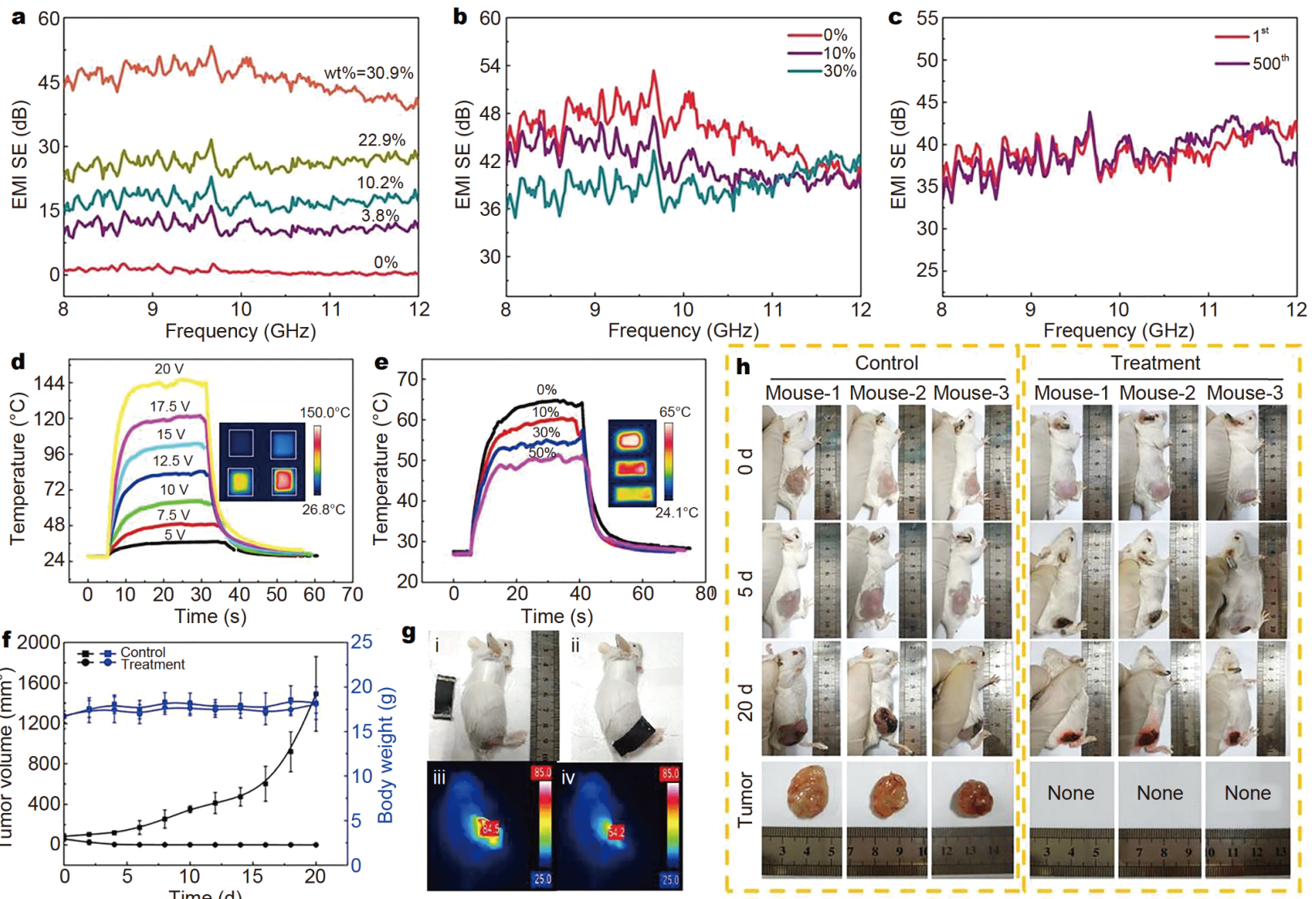

Frequency $(\mathrm{GHz})$

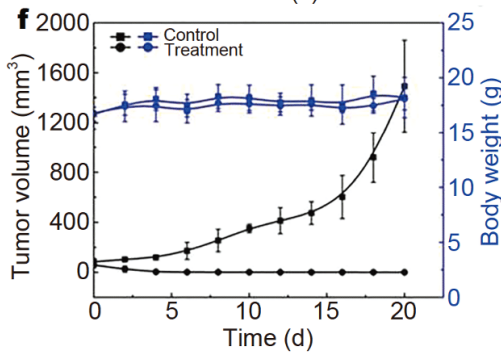

$\mathbf{g}$
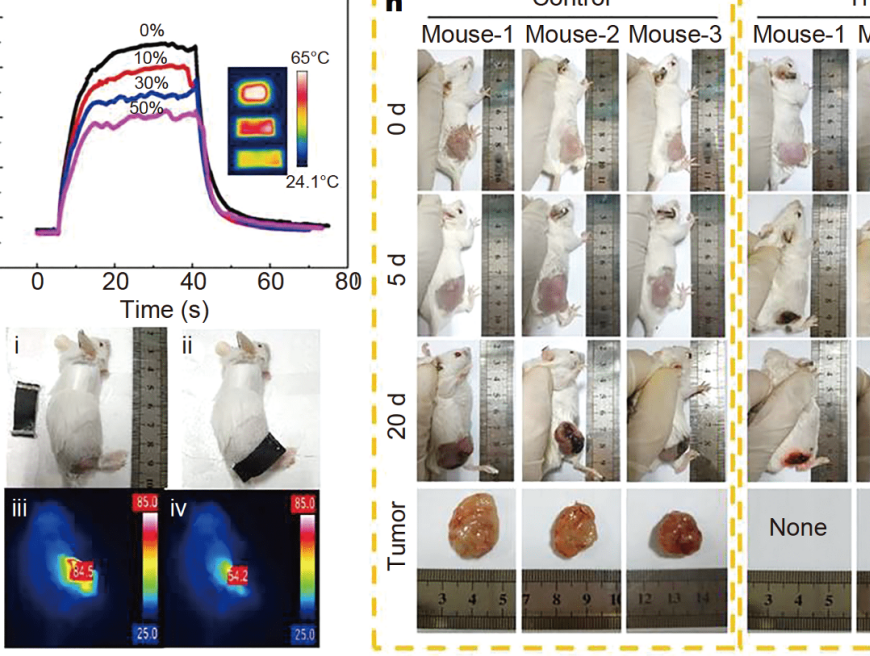

Figure 6 Electromagnetic shielding properties of $\mathrm{BSPF}_{m}$ at $8-12 \mathrm{GHz}$ and electro-thermal tumor removal using BSPF ${ }_{m}$. (a) EMI SE as a function of frequency for BSPF $_{m}$ having different SWCNT contents. (b) EMI SE as a function of frequency for $\mathrm{BSPF}_{7}$ at different applied strains. (c) The EMI SE of the $\mathrm{BSPF}_{7}$ after 500 cycles at $30 \%$ tensile strain. (d) Temperature as a function of time for $\mathrm{BSPF}_{5}$ at different applied voltages. The insets were thermal images at $5,10,15$, and $20 \mathrm{~V}$. (e) Temperature as a function of time for $\mathrm{BSPF}_{5}$ at different applied strains under $10 \mathrm{~V}$ voltage. The insets were thermal images at strains of $0 \%, 30 \%$, and $50 \%$. (f) Tumor volume and body weight as a function of time for the tumor-removal mice by thermal ablation and for the control mice. (g) Optical images before (i) and during (ii) thermal ablation of tumor tissue using $B_{S P F_{5}}$ and thermal images of tumor tissue covered with (iii) and uncovered with (iv) $\mathrm{BSPF}_{5}$ during thermal ablation. (h) Photographs showed the tumor tissue grown in the control mice in 20 days (left) and removed in treatment mice in 20 days (right). 
large deformation, we here demonstrated the use of a biaxial $\mathrm{BSPF}_{5}$ as an electrode for electro-thermal tumor removal. An advantage of using $\mathrm{BSPF}_{m}$ for heating is that the electricity can be applied on one side and the opposite side can also be heated due to the bulk conductivity. This would facilitate the operation during surgery. A biaxial $\mathrm{BSPF}_{5}$ with a dimension of $1.0 \mathrm{~cm} \times 1.1 \mathrm{~cm} \times 0.15 \mathrm{~mm}$ was used in this study, and its electro-heating properties under different applied voltages and different applied strains were first investigated. The temperature of the $\mathrm{BSPF}_{5}$ reached a plateau of $84.5^{\circ} \mathrm{C}$ within $18 \mathrm{~s}$ by applying a voltage of $12.5 \mathrm{~V}$, and decreased in $13 \mathrm{~s}$ upon removal of the voltage (Fig. 6d). The plateau temperature increased from 37 to $140^{\circ} \mathrm{C}$ by increasing the voltage from 5 to $20 \mathrm{~V}$, indicating that temperature can be easily controlled by using different voltages. Stretching the $\mathrm{BSPF}_{5}$ from $0 \%$ to $50 \%$ strain with $10 \mathrm{~V}$ of voltage decreased the plateau temperature from 64 to $50^{\circ} \mathrm{C}$ (Fig. 6e), which is high enough for tumor removal. This high flexibility would enable good contact of the electrode with irregular tumor surface. Thermal images in the insets in Fig. $6 \mathrm{~d}$ and e indicate uniform temperature distribution on the $\mathrm{BSPF}_{5}$ surface, which is important for the operation in tumor removal.

We then investigated the in vivo electro-thermal tumor removal using $\mathrm{BSPF}_{5}$ on Hepa1-6-tumor-bearing mice, with the tumor tissue embedded below the skin of abdomen (Fig. $6 \mathrm{f}-\mathrm{h}$ ). The experimental details refer to the Supplementary information. All animal procedures were performed according to the protocol approved by the Institutional Animal Care and Use Committee at China Pharmaceutical University (Permit Number: CCPU 2018056). A BSPF 5 with size of $1.2 \mathrm{~cm} \times 2.5 \mathrm{~cm} \times 0.15 \mathrm{~mm}$ was connected with two copper wires on both ends using silver paste. The $\mathrm{BSPF}_{5}$ was wrapped over the tumor (Fig. $6 \mathrm{~g}(\mathrm{ii})$ ), and a $12.5 \mathrm{~V}$ voltage was applied for heating for $30 \mathrm{~min}$, corresponding to a power density of $0.26 \mathrm{~W} \mathrm{~cm}^{-2}$. The temperature of the top surface of $\mathrm{BSPF}_{5}$ increased to $84.5^{\circ} \mathrm{C}$ in $3 \mathrm{~min}$ and kept at this temperature for $30 \mathrm{~min}$ to heat the tumor tissue (Fig. $6 \mathrm{~g}$ (iii)). And the tumor surface was at a temperature of $54.2^{\circ} \mathrm{C}$ during treatment (Fig. $6 \mathrm{~g}(\mathrm{iv})$ ). The electro-thermal treatment was repeated for another two times every five days. The mice were weighed every day after tumor ablation to monitor the health conditions, and the tumor size was also measured every day after treatment. It was shown that there was negligible change in body weight for all mice (Fig. 6f). For the tumor-removal mice, the tumor size decreased gradually and even entirely disappeared, and no recurrence of tumor was observed (Fig. $6 \mathrm{f}$ and h, right). As a comparison, the control experiments showed that the tumor-bearing mice showed increased tumor size after 20 days of observation (Fig. $6 \mathrm{f}$ and $\mathrm{h}$, left). The cell viability was tested on $\mathrm{C} 2 \mathrm{C} 12$ cells by using (3-(4,5-dimethylthiazol-2-yl)-2,5-diphenyltetrazolium bromide) tetrazolium (MTT) assay, terminal deoxynucleotidyl transferase-mediated dUTP-digoxigenin nick-end labeling (TUNEL) assay, and cell attachment. The $\mathrm{BSPF}_{5}$ showed low in vitro cell toxicity (Figs S27 and S28), indicating high biological safety when the $\mathrm{BSPF}_{m}$ were used for tumor therapy.

\section{CONCLUSIONS}

Buckled structures were introduced into the 3D conductive network of an intrinsic elastic conductor, enabling uniform bulk conductivity and stable resistance under large deformation. This was realized by self-assembly of SWCNT/PVP dispersion on the interconnecting pore surface of a pre-strained PP foam (to prepare the conductive network), and strain relaxing to enable buckle formation. The resulting buckled intrinsic conductor showed resistance variation as low as $2.0 \%$ for $70 \%$ strain and a quality factor as low as 0.028 . Both unfolding of buckles and flattening of micropores contributed to the stability of the resistance under deformation. Microstructural analysis illustrated different patterns of $2 \mathrm{D}$ buckling structures, as simulated by FEA, which is due to the imperfections in the conductive layer. These properties enabled their applications as all-directional interconnects, EMI shielding, and tumor ablation electrodes. Our strategy could be applied to other polymer matrices (including PU, PDMS, and natural rubber) and other conductive fillers (including metal nanowires and nanoparticles, graphene, and MXene) to improve the electrical and mechanical properties, as well as incorporating other functionalities. This would expand their applications for wearable electronics and smart robotics.

Received 12 January 2020; accepted 12 February 2020; published online 1 April 2020

1 Hwang BU, Lee JH, Trung TQ, et al. Transparent stretchable selfpowered patchable sensor platform with ultrasensitive recognition of human activities. ACS Nano, 2015, 9: 8801-8810

2 Jeong CK, Lee J, Han S, et al. A hyper-stretchable elastic-composite energy harvester. Adv Mater, 2015, 27: 2866-2875

3 Kim KK, Hong S, Cho HM, et al. Highly sensitive and stretchable multidimensional strain sensor with prestrained anisotropic metal nanowire percolation networks. Nano Lett, 2015, 15: 5240-5247

4 Han S, Hong S, Ham J, et al. Fast plasmonic laser nanowelding for a Cu-nanowire percolation network for flexible transparent conductors and stretchable electronics. Adv Mater, 2014, 26: 5808- 
5814

$5 \mathrm{Xu}$ F, Zhu Y. Highly conductive and stretchable silver nanowire conductors. Adv Mater, 2012, 24: 5117-5122

6 Hansen $\mathrm{T}$, West $\mathrm{K}$, Hassager $\mathrm{O}$, et al. Highly stretchable and conductive polymer material made from poly(3,4-ethylenedioxythiophene) and polyurethane elastomers. Adv Funct Mater, 2007, 17: 3069-3073

7 Lee YY, Kang HY, Gwon SH, et al. A strain-insensitive stretchable electronic conductor: PEDOT:PSS/acrylamide organogels. Adv Mater, 2016, 28: 1636-1643

8 Markvicka EJ, Bartlett MD, Huang X, et al. An autonomously electrically self-healing liquid metal-elastomer composite for robust soft-matter robotics and electronics. Nat Mater, 2018, 17: 618-624

9 Yun G, Tang SY, Sun S, et al. Liquid metal-filled magnetorheological elastomer with positive piezoconductivity. Nat Commun, 2019, 10: 1300

10 Chun KY, Oh Y, Rho J, et al. Highly conductive, printable and stretchable composite films of carbon nanotubes and silver. Nat Nanotech, 2010, 5: 853-857

11 Matsuhisa N, Kaltenbrunner M, Yokota T, et al. Printable elastic conductors with a high conductivity for electronic textile applications. Nat Commun, 2015, 6: 7461

12 Hong S, Lee H, Lee J, et al. Highly stretchable and transparent metal nanowire heater for wearable electronics applications. Adv Mater, 2015, 27: 4744-4751

13 Jung J, Lee H, Ha I, et al. Highly stretchable and transparent electromagnetic interference shielding film based on silver nanowire percolation network for wearable electronics applications. ACS Appl Mater Interfaces, 2017, 9: 44609-44616

14 Lipomi DJ, Lee JA, Vosgueritchian M, et al. Electronic properties of transparent conductive films of PEDOT:PSS on stretchable substrates. Chem Mater, 2012, 24: 373-382

15 Vosgueritchian M, Lipomi DJ, Bao Z. Highly conductive and transparent PEDOT:PSS films with a fluorosurfactant for stretchable and flexible transparent electrodes. Adv Funct Mater, 2012, 22: $421-428$

16 Yao S, Zhu Y. Nanomaterial-enabled stretchable conductors: Strategies, materials and devices. Adv Mater, 2015, 27: 1480-1511

17 Bartlett MD, Markvicka EJ, Majidi C. Rapid fabrication of soft, multilayered electronics for wearable biomonitoring. Adv Funct Mater, 2016, 26: 8496-8504

18 Kim DH, Lu N, Ma R, et al. Epidermal electronics. Science, 2011, 333: 838-843

19 Jeong H, Wang L, Ha T, et al. Modular and reconfigurable wireless E-tattoos for personalized sensing. Adv Mater Technol, 2019, 4: 1900117

20 Kim A, Ahn J, Hwang H, et al. A pre-strain strategy for developing a highly stretchable and foldable one-dimensional conductive cord based on a Ag nanowire network. Nanoscale, 2017, 9: 5773-5778

21 Lee $\mathrm{P}$, Lee J, Lee $\mathrm{H}$, et al. Highly stretchable and highly conductive metal electrode by very long metal nanowire percolation network. Adv Mater, 2012, 24: 3326-3332

22 Liu ZF, Fang S, Moura FA, et al. Hierarchically buckled sheathcore fibers for superelastic electronics, sensors, and muscles. Science, 2015, 349: 400-404

23 Zhu Y, Xu F. Buckling of aligned carbon nanotubes as stretchable conductors: A new manufacturing strategy. Adv Mater, 2012, 24: 1073-1077

24 Greco F, Fujie T, Ricotti L, et al. Microwrinkled conducting polymer interface for anisotropic multicellular alignment. ACS Appl Mater Interfaces, 2013, 5: 573-584

25 Trung TQ, Lee NE. Recent progress on stretchable electronic devices with intrinsically stretchable components. Adv Mater, 2017, 29: 1603167

26 Liu Z, Wan F, Mou L, et al. A general approach for buckled bulk composites by combined biaxial stretch and layer-by-layer deposition and their electrical and electromagnetic applications. Adv Electron Mater, 2019, 5: 1800817

27 Weng C, Dai Z, Wang G, et al. Elastomer-free, stretchable, and conformable silver nanowire conductors enabled by three-dimensional buckled microstructures. ACS Appl Mater Interfaces, 2019, 11: 6541-6549

28 O'Connell MJ, Boul P, Ericson LM, et al. Reversible water-solubilization of single-walled carbon nanotubes by polymer wrapping. Chem Phys Lett, 2001, 342: 265-271

29 Thomas AV, Andow BC, Suresh S, et al. Controlled crumpling of graphene oxide films for tunable optical transmittance. Adv Mater, 2015, 27: 3256-3265

30 Yu Y, Zeng J, Chen C, et al. Three-dimensional compressible and stretchable conductive composites. Adv Mater, 2014, 26: 810-815

31 Ge J, Yao HB, Wang X, et al. Stretchable conductors based on silver nanowires: improved performance through a binary network design. Angew Chem Int Ed, 2013, 52: 1654-1659

32 Wu C, Fang L, Huang X, et al. Three-dimensional highly conductive graphene-silver nanowire hybrid foams for flexible and stretchable conductors. ACS Appl Mater Interfaces, 2014, 6: 21026-21034

33 Duan S, Wang Z, Zhang L, et al. Three-dimensional highly stretchable conductors from elastic fiber mat with conductive polymer coating. ACS Appl Mater Interfaces, 2017, 9: 30772-30778

34 Tang Y, Gong S, Chen Y, et al. Manufacturable conducting rubber ambers and stretchable conductors from copper nanowire aerogel monoliths. ACS Nano, 2014, 8: 5707-5714

35 Kim KH, Vural M, Islam MF. Single-walled carbon nanotube aerogel-based elastic conductors. Adv Mater, 2011, 23: 2865-2869

36 Gao HL, Xu L, Long F, et al. Macroscopic free-standing hierarchical 3D architectures assembled from silver nanowires by ice templating. Angew Chem Int Ed, 2014, 53: 4561-4566

37 Oh JY, Lee D, Hong SH. Ice-templated bimodal-porous silver nanowire/PDMS nanocomposites for stretchable conductor. ACS Appl Mater Interfaces, 2018, 10: 21666-21671

38 Chen Z, Ren W, Gao L, et al. Three-dimensional flexible and conductive interconnected graphene networks grown by chemical vapour deposition. Nat Mater, 2011, 10: 424-428

39 Chortos A, Liu J, Bao Z. Pursuing prosthetic electronic skin. Nat Mater, 2016, 15: 937-950

40 Lipomi DJ, Vosgueritchian M, Tee BCK, et al. Skin-like pressure and strain sensors based on transparent elastic films of carbon nanotubes. Nat Nanotech, 2011, 6: 788-792

41 Mates JE, Bayer IS, Palumbo JM, et al. Extremely stretchable and conductive water-repellent coatings for low-cost ultra-flexible electronics. Nat Commun, 2015, 6: 8874

42 Chen Z, Xu C, Ma C, et al. Lightweight and flexible graphene foam composites for high-performance electromagnetic interference shielding. Adv Mater, 2013, 25: 1296-1300

43 Wan YJ, Zhu PL, Yu SH, et al. Anticorrosive, ultralight, and flexible carbon-wrapped metallic nanowire hybrid sponges for highly efficient electromagnetic interference shielding. Small, 2018, 14: 1800534 
Chu KF, Dupuy DE. Thermal ablation of tumours: biological mechanisms and advances in therapy. Nat Rev Cancer, 2014, 14: 199-208

45 Sotiriou GA, Starsich F, Dasargyri A, et al. Photothermal killing of cancer cells by the controlled plasmonic coupling of silica-coated $\mathrm{Au} / \mathrm{Fe}_{2} \mathrm{O}_{3}$ nanoaggregates. Adv Funct Mater, 2014, 24: 2818-2827 Zhou Y, Wang R, Teng Z, et al. Magnetic nanoparticle-promoted droplet vaporization for in vivo stimuli-responsive cancer theranostics. NPG Asia Mater, 2016, 8: e313

47 Svirskis D, Travas-Sejdic J, Rodgers A, et al. Electrochemically controlled drug delivery based on intrinsically conducting polymers. J Control Release, 2010, 146: 6-15

Acknowledgements This work was supported by the National Key Research and Development Program of China (2017YFB0307000), the National Natural Science Foundation of China (51973093, U1533122 and 51773094), the Natural Science Foundation of Tianjin (18JCZDJC36800), the Science Foundation for Distinguished Young Scholars of Tianjin (18JCJQJC46600), the Fundamental Research Funds for the Central Universities (63171219), the State Key Laboratory for Modification of Chemical Fibers and Polymer Materials, Donghua University (LK1704), the National Special Support Plan for High-level Talents people (C041800902), and the Eugene McDermott Graduate Fellows Program.

Author contributions Liu ZF was responsible for the experimental concept and design. He W, Cheng Y, Zhou X, Hu X, Liu ZS, Sun J and Wang Y carried out the most of the experiments, characterization and data analyses. Zhang R, Qian D and Liu ZJ contributed to the theoretical simulation and calculation. Zhang $\mathrm{C}$ carried out the cell viability experiments and data analyses. All authors contributed to the general discussion. All authors provided comments and agreed with the final form of the manuscript.

Conflict of interest The authors declare that they have no conflict of interest.

Supplementary information Supplementary material for this article is available in the online version of the paper.

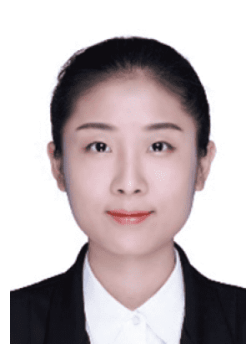

Wenqian He is currently a MSc candidate under the supervision of Prof. Zunfeng Liu at the State Key Laboratory of Medicinal Chemical Biology, Nankai University. Her research interest focuses on the intrinsic elastic conductor for flexible wearable electronics.

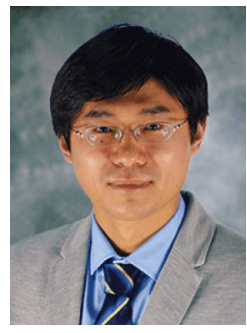

Zunfeng Liu is a professor at the Key Laboratory of Functional Polymer Materials, College of Pharmacy and the State Key Laboratory of Medicinal Chemical Biology, Nankai University. He received his $\mathrm{PhD}$ from Nankai University in 2008. From 2008 to 2012, he was working as a postdoc fellow and project leader in Erasmus Medical Center, Leiden University (Netherlands). From 2013 to 2016, he was a professor at Changzhou University. His research expertise is in the area of wearable electronics, artificial muscles and actuators, especially in developing highly stretchable electronic interconnects, sensors and medical devices.

\section{具有祤皱导电路径的本征体弹性导体用于柔性电 磁屏蔽和肿瘤消融}

何文倩 ${ }^{1}$, 张锐 ${ }^{2}$, 程媛媛 ${ }^{3}$, 张超 ${ }^{4}$, 周湘 ${ }^{4}$, 刘壮健 ${ }^{5}$, 胡晓宇 ${ }^{1}$, 刘中胜 ${ }^{1}$, 孙进坤 ${ }^{1}$, 王银松 ${ }^{3}$, 钱冬 ${ }^{2}$, 刘遵峰 ${ }^{{ }^{*}}$

摘要 弹性导体在可穿戴电子设备和软机器人中至关重要. 理想的 本征弹性体导体在大形变下显示出均匀的三维导电网络结构和稳 定的电阻变化. 其面临的一个挑战是, 由于体弹性导体在形变下导 电路径断开, 电阻变化很大. 我们的策略是将具有互连微孔的泡沫 进行预拉伸, 通过碳纳米管在微孔表面自组装形成导电层, 随后释 放预应变, 从而在导电网络中引入褶皱结构. 在拉伸过程中, 褶皱和 微孔的变形都有助于电阻的稳定(在70\%应变下电阻变化 $2.0 \%$ ). 微 观结构和有限元分析表明, 由于导电层中的缺陷, 可以获得不同形 貌的二维褶皱结构. 我们展示了其在全方位电气互连、可拉伸电 磁屏蔽和电热肿瘤消融方面的应用. 